\title{
La mise en tourisme du patrimoine viticole : l'œnotourisme dans les vignobles du Cap Bon (Tunisie)
}

Tourism Development of Wine Heritage: Wine Tourism in the Vineyards of Cap Bon (Tunisia)

\section{Mohamed Souissi}

\section{OpenEdition} Journals

Édition électronique

URL : https://journals.openedition.org/tourisme/3575

DOI : $10.4000 /$ tourisme.3575

ISSN : 2492-7503

\section{Éditeur}

Association Mondes du tourisme

\section{Référence électronique}

Mohamed Souissi, «La mise en tourisme du patrimoine viticole : l'œnotourisme dans les vignobles du Cap Bon (Tunisie) », Mondes du Tourisme [En ligne], 19 | 2021, mis en ligne le 15 septembre 2021, consulté le 17 septembre 2021. URL : http://journals.openedition.org/tourisme/3575 ; DOI : https:// doi.org/10.4000/tourisme.3575

Ce document a été généré automatiquement le 17 septembre 2021.

\section{cc)}

Mondes du tourisme est mis à disposition selon les termes de la licence Creative Commons Attribution - Pas d'Utilisation Commerciale - Pas de Modification 4.0 International. 


\title{
La mise en tourisme du patrimoine viticole : l'œnotourisme dans les vignobles du Cap Bon (Tunisie)
}

\author{
Tourism Development of Wine Heritage: Wine Tourism in the Vineyards of \\ Cap Bon (Tunisia)
}

Mohamed Souissi

\section{Introduction}

1 Les vignobles, dans le monde, représentent aujourd'hui à la fois une activité agricole, un enjeu économique valorisé par des paysages, un patrimoine, une ressource touristique et un produit de terroir ${ }^{1}$. La viticulture s'associe au tourisme pour enrichir le produit et l'offre culturelle du tourisme local en valorisant les paysages viticoles, les chais et musées du vin s'ouvrent désormais à des visiteurs de plus en plus nombreux (Lignon-Darmaillac, 2014). L'œnotourisme, qui est une forme d'agritourisme ${ }^{2}$, se trouve à l'intersection du tourisme et du territoire viticole. Par sa répartition géographique, il diffère de toutes les formes conventionnelles de tourisme issues de la massification des pratiques qui se développent dans des resorts fermés et s'appuient exclusivement sur la ressource balnéaire sans réelle considération a priori pour le patrimoine local. Par son côté social, le touriste est en contact direct avec les habitants de la région viticole visitée. Le retour aux valeurs fortes que représentent la campagne et son terroir viticole, à savoir son authenticité ${ }^{3}$ et sa convivialité, ainsi que la recherche d'un cadre de vie qui allie harmonieusement paysage, environnement, patrimoine, production agricole et gastronomie locale, sont les motivations premières des adeptes de cette forme de tourisme. La désaffection des lieux et destinations touristiques très fréquentés pendant les périodes de hautes saisons touristiques est également un élément motivant pour se tourner vers un tourisme différent de celui des grandes stations balnéaires.

2 Les ressources et les potentialités touristiques du territoire viticole, ses paysages, ses patrimoines et l'effort important mis en œuvre par l'ensemble des acteurs pour 
proposer des hébergements de qualité et des animations variées, ajoutés à la chaleur et à la qualité de "l'accueil paysan", font que les vignobles deviennent une destination attractive. La démarche de reconnaissance du patrimoine viticole comme ressource touristique est de nature socioculturelle : au-delà du fait qu'elle implique l'engagement de toute la population concernée, directement ou indirectement, elle commence par l'initiative d'un individu ou d'un groupe d'individus, appuyés par une population locale voire régionale (Pichery, 2018).

3 La Tunisie, en crise touristique depuis « la révolution de Jasmin » de 2011 - malgré une reprise des fréquentations internationales (hors Maghreb) en 2018 -, recherche des alternatives au tourisme conventionnel centré sur la ressource balnéaire. Les terroirs viticoles de la péninsule du Cap Bon, au nord-est de la Tunisie, se révèlent être des espaces particulièrement adéquats pour développer d'autres formes de tourisme alternatif ${ }^{4}$. Le fait que les territoires ruraux soient actuellement recherchés pour leur riche potentiel touristique (patrimoine naturel, viticole et historique), comme alternative au tourisme conventionnel, situe ces terroirs viticoles en excellente position pour associer territoire, paysage et patrimoine culturel. La possibilité d'intégrer la dimension paysagère et patrimoniale dans les activités touristiques et la mise en tourisme des petits patrimoines et des ressources naturelles non liées exclusivement au soleil et à la mer trouvent ainsi un nouveau sens (produits de terroirs, routes viticoles).

4 Notre investigation consiste à comprendre le fonctionnement de l'œnotourisme et à montrer dans quelles conditions le territoire rural du Cap Bon pourrait intégrer la valorisation de son paysage et de son patrimoine viticole dans les politiques de diversification du produit touristique tunisien. Nos travaux sur la mise en tourisme du patrimoine viticole reposent sur un regard croisé entre la géographie du tourisme et la géographie culturelle, dans un processus de patrimonialisation des espaces ruraux et des produits dits de terroir. Notre méthodologie s'appuie sur des études de cas induisant des réflexions de portée générale. Cette méthodologie, soutenue par des approches prospectives, est développée à travers une démarche émanant d'un travail de terrain fondé sur des entretiens semi-directifs et des questionnaires. Au cours de nos travaux, nous nous sommes rendu à plusieurs reprises dans les vignobles du Cap Bon, en 2016 et en 2017, pour y étudier le développement de l'œnotourisme dans les fermes viticoles. Afin de mieux appréhender le fonctionnement de l'œnotourisme, nous avons, d'une part, réalisé 24 entretiens auprès de différentes catégories d'acteurs: propriétaires de caves coopératives et d'entreprises vinicoles, guides-animateurs, vignerons, population locale. Nous avons, d'autre part, administré un questionnaire à 43 visiteurs des fermes viticoles afin de mieux comprendre le profil des visiteurs et les enjeux de la demande œnotouristique.

5 Analyser la relation entre le tourisme et les potentialités viticoles permet de comprendre que l'œnotourisme est l'un des produits de tourisme alternatif qui pourrait contribuer à la valorisation touristique des terroirs viticoles du Cap Bon ainsi que des territoires qui le composent. Ce nouveau produit pourrait participer, à l'avenir, au développement économique des régions concernées. La lecture des différents travaux de recherche et d'enquête sur le tourisme viticole ainsi que la rencontre des acteurs locaux et des visiteurs des fermes viticoles ont permis de saisir l'état des lieux de l'œnotourisme dans les territoires ruraux du Cap Bon et de mesurer ses enjeux et ses 
perspectives de développement à l'échelle de la péninsule et à l'échelle de chaque terroir portant un potentiel touristique spécifique.

\section{L'œnotourisme : concept et cadrage théorique}

\subsection{L'œnotourisme ou tourisme viticole : une valorisation des produits et du patrimoine viticoles}

Olivier Lazzarotti $(2003,2011)$ présente la relation entre tourisme et patrimoine comme une relation de complémentarité. Ce sont deux phénomènes sociaux mis en liaison par leurs acteurs. Loin de s'opposer, comme il est souvent dit, tourisme et patrimoine se valorisent et se renforcent réciproquement et, de plus en plus, inséparablement. Ils sont activés ensemble pour être orientés vers un but commun. Ils servent, en quelque sorte, d'instruments d'aménagement de l'espace et de promotion du lieu (Lazzarotti, 2003). Pour être valide, le patrimoine a besoin du regard de l'autre, donc du tourisme qui en fait très largement figure. Le tourisme apparait ainsi comme porteur de patrimoine, autant que le patrimoine appelle le tourisme. Le patrimoine est ainsi lié à la pratique des lieux et à la mobilité des touristes. La mise en tourisme se fait par réactivation d'éléments patrimoniaux (Lazzarotti, 2011).

7 L'œnotourisme est un terme à la mode, utilisé dans les médias et très sollicité aujourd'hui dans le discours touristique francophone. Il engendre un flou conceptuel, d'autant qu'œnotourisme et tourisme viticole sont parfois confondus. En réalité, le tourisme viticole émane plutôt des viticultures alors que l'œnotourisme est une pratique plus récente et davantage portée par les acteurs touristiques et politiques. Dans le sud de l'Angleterre et au Pays de Galles, par exemple, le tourisme viticole est pratiqué par la majorité des viticultures, qui tirent ainsi le meilleur bénéfice possible de leur production (Pitte, 1988). De même, dans le canton du Valais suisse, le tourisme et la vigne sont considérés comme deux secteurs importants de l'économie et de la vie valaisanne: les amateurs de vin visitent les vignobles et les caves dans les régions produisant des crus reconnus, et le contact direct avec des vignerons et des encaveurs est particulièrement prisé. Les professionnels du tourisme se sont rapprochés des viticulteurs et de la population locale afin de les faire participer à la création d'une offre touristique destinée à attirer les vacanciers (Raboud-Schüle, 1993).

Sophie Lignon-Darmaillac définit l'œnotourisme comme «réunissant l'ensemble de toutes les activités touristiques, de loisirs et de temps libre dédiées à la découverte et à la jouissance culturelle et œnophile de la vigne, du vin et de son terroir» (2009, p 21). Cette définition, si elle donne un cadre en tant qu'activité touristique, ne permet guère de définir précisément les activités et les pratiques effectuées par les touristes. Elle présente l'avantage de considérer l'œnotourisme en tant qu'une forme de tourisme qui repose sur l'attrait du patrimoine construit par les sociétés rurales spécialisées dans la viticulture, où le divertissement des visiteurs passe par la pratique d'activités de loisirs dans un espace viticole. Cette définition peut également être mise en perspective avec celle de la Charte européenne de l'œnotourisme qui définit l'œnotourisme comme «le développement de toutes les activités touristiques et de temps libre dédiées à la découverte et à la jouissance culturelle et œnophile de la vigne, du vin et de son territoire » (VINTUR, 2005, p 4). La Charte exprime la volonté des acteurs impliqués et des professionnels du tourisme et de la viticulture de favoriser un œnotourisme 
conforme aux principes du développement durable. Le développement, ainsi prévu, nécessite le renforcement de toutes les interactions positives entre l'activité œnotouristique (secteur vitivinicole et touristique) et les autres secteurs économiques d'un territoire. Cette définition, à son tour, renvoie au rapport Dubrule de 2007 qui tranche la question du terme et qui fixe et définit l'œnotourisme en France comme "l'ensemble des prestations relatives aux séjours des touristes dans des régions viticoles : visites de caves, dégustations, hébergement, restauration et activités annexes liées au vin, aux produits du terroir et aux traditions régionales » (Dubrule, 2007, p 6). Il place ainsi l'œnotourisme dans le double champ de l'économie touristique et de l'économie viticole. À partir de ce rapport, le terme d'œnotourisme et son concept vont se diffuser largement dans le discours touristique francophone. Il répond en effet à une demande sociétale en faveur des produits de qualité, des terroirs, de la typicité des paysages, et fait converger les acteurs du tourisme et les pouvoirs publics vers le monde viticole.

9 Afin de proposer une offre complète, les activités et les pratiques de l'œnotourisme s'appuient sur une association forte entre les patrimoines viticoles et les autres patrimoines locaux, et participent ainsi à la dynamique de patrimonialisation ${ }^{5}$ au sein du territoire (Lemarié-Boutry, 2016). Les acteurs locaux doivent dès lors s'approprier des ressources locales spécifiques, naturelles ou culturelles. On rejoint ici le concept de création d'un "panier de biens et services ", dans lequel «l'histoire et la culture du lieu pèsent davantage sur la caractérisation de l'image du panier de biens pour le consommateur " (Pecqueur, 2001, p. 43). Le patrimoine, viticole ou non, constitue dans ce panier une ressource spécifique, résultat d'une construction des acteurs. L'œnotourisme semble donc s'appuyer sur une sélection et une réinvention $d u$ patrimoine, vecteur de l'identité des acteurs qui le recomposent.

10 Les vignobles deviennent les destinations d'un tourisme culturel fondé sur de riches patrimoines paysagers et architecturaux et façonnés par les sociétés vigneronnes. L'œnotourisme met en lumière le monde viticole, patrimonialise les paysages et les traditions vigneronnes et fait naitre de nouvelles formes de patrimoines ruraux. Les paysages viticoles présentent ainsi une dimension patrimoniale aujourd'hui reconnue par la communauté internationale. Résultant de travaux d'aménagement commencés par les générations précédentes, leur transmission aux générations futures s'est imposée en raison de leurs valeurs mémorielles. Depuis 1992, la catégorie des paysages culturels a ainsi été introduite dans la Liste du patrimoine mondial de l'Unesco, où la vigne joue un rôle majeur. Actuellement, onze paysages culturels inscrits sur la Liste du patrimoine mondial sont concernés, qu'ils soient entièrement viticoles ou qu'ils comprennent une part importante de vignes; ils se trouvent tous en Europe. Et, sur la liste indicative, aux côtés du dossier du Piémont, en cours d'instruction, et de ceux achevés et déposés des climats de Bourgogne et des coteaux, caves et maisons de Champagne, sept autres vignobles figurent également, dont un seul non européen, celui du Cap (Afrique du Sud).

11 Le fort intérêt porté au patrimoine viticole se traduit non seulement par la valorisation des paysages, mais aussi par la reconnaissance et la mise en tourisme du patrimoine architectural des caves, des chais, des maisons de négoce, autant d'exploitations et de fermes viticoles répertoriées sur les routes des vins comme opportunités de lieux à visiter. Ces exploitations sont le plus souvent des bâtiments traditionnels intégrés aux normes architecturales locales. Ces bâtiments font aujourd'hui appel aux plus grands 
architectes, de renommée internationale : constructions futuristes de Frank O. Ghery (Marqués de Riscal à Elciego), Zaha Hahid (Lopéz de Heredia à Haro) ou, en France, Xavier Leibar (château Thuerry) et Jean Nouvel (château La Coste) en Provence... Ces architectures, fortement personnalisées, ouvrent ces propriétés à des clientèles nouvelles qui, parfois sans rien connaitre au monde viticole, recherchent l'originalité de cette nouvelle alliance du vin et de l'art contemporain sous toutes ses formes (Lignon-Darmaillac, 2015).

12 En tant qu'outil de découverte du patrimoine viticole, la route viticole ${ }^{6}$ permet de relier et de valoriser les différents patrimoines paysagers et architecturaux présents dans les vignobles. La route des vins met en lien les divers matériaux qui constituent le patrimoine. Elle permet de mettre en évidence les limites du terroir viticole ainsi que ce qui participe de son unité, à savoir le patrimoine matériel des bourgs, des bâtiments agricoles et des paysages, la gastronomie et le patrimoine immatériel des savoir-faire et de la culture locale des villages spécialisés depuis des décennies dans la production du vin. Les routes des vins offrent l'opportunité de déguster, non seulement dans les caves, mais aussi dans une grande diversité de lieux, selon des offres de plus en plus diversifiées. Des routes se sont ainsi intégrées à des itinéraires plus complexes, réseaux d'adresses de lieux de visite et de consommation, dont le vignoble est le cadre privilégié, que l'on découvre par la route et les sentiers vignerons. Ils conduisent ainsi à un tourisme œnogastronomique pour lequel les offres de dégustation de vins sont de plus en plus variées, partagées entre caves, tables d'hôtes et restaurants (LignonDarmaillac, 2019). Si les routes des vins les plus anciennes ont été inaugurées en Europe à la fin des années 1930 (comme la route des Grands Crus en Bourgogne), puis dans les années 1950 (en Alsace et en Champagne), c'est depuis la dernière décennie du $\mathrm{xx}^{\mathrm{e}}$ siècle qu'elles se sont multipliées partout dans le monde. En Allemagne, en Bourgogne, puis de nouveau en France en Alsace et en Champagne, plus tardivement en Afrique du Sud, des routes ont permis de reconnaitre la dimension touristique de grands vignobles. La France, par exemple, compte plus de 300 routes touristiques à thème ${ }^{7}$. Les vignobles ont évidemment la cote. Parmi les dizaines d'itinéraires qui leur sont consacrés, citons la route des vins d'Alsace, la route des vins Mâconnais-Beaujolais (Bourgogne du Sud), la route touristique des vignobles du Cœur de France (Val de Loire), la route des vins (Provence) et la route touristique du Champagne. Les routes historiques ont permis de développer les ventes directes, de fidéliser la clientèle. Elles se sont définies, peu à peu, comme un véritable outil de promotion et de marketing pour la filière viticole (Beaudet, 2003).

13 Ainsi, l'œnotourisme regroupe des activités et des pratiques très diverses qui peuvent avoir pour intérêt une pluralité de thématiques reliées à la notion de patrimoine et correspondant à l'ensemble des prestations relatives aux séjours des touristes dans des régions viticoles: visites de caves et de musées ${ }^{8}$, dégustations, hébergement, restauration et activités annexes liées au vin, aux produits du terroir et aux traditions régionales. La route viticole associée à la découverte du patrimoine gastronomique ${ }^{9}$ est ainsi au cœur de la pratique de l'œnologie, de la dégustation des produits locaux dans les domaines viticoles. Les pratiques des vendanges, la découverte des techniques de la vigne et du vin participent également de la valorisation du patrimoine immatériel viticole.

14 Pour appuyer cet essai de cadrage théorique de l'œnotourisme, nous analyserons le cas de Chianti, en Italie, qui illustre la nécessité d'articuler les compétences d'acteurs 
locaux d'horizons divers pour créer des prestations œnotouristiques qui associent le vin à d'autres aménités locales, entraînant ainsi la valorisation du patrimoine viticole et le développement d'une dynamique touristique plus vaste.

\subsection{L'exemple de Chianti en Italie ${ }^{10}$ : premier espace viticole européen à avoir été mis en tourisme ${ }^{11}$}

L'œnotourisme est une forme de tourisme qui s'organise autour de la thématique du vin et de son patrimoine avec des itinéraires permettant de se promener parmi les champs de vignes et le patrimoine bâti qui leur est associé. Le Chianti Classico est un terroir italien dont le vin, comme le patrimoine viticole, sont renommés à l'échelle internationale. Le tourisme viticole y est très développé et connaît une croissance constante depuis plusieurs années, aussi bien en termes d'offre que de demande. Situé entre Florence et Sienne, le vignoble de Chianti ${ }^{12}$ est un des pôles touristiques majeurs de la Toscane, de renommée internationale. Les étrangers s'y rendent nombreux, attirés par ses paysages de collines méditerranéennes et par la renommée croissante de son vin rouge, autour de l'appellation phare Chianti Classico. La vigne y est cultivée depuis l'Antiquité. Les premiers relevés parcellaires datent de la fin du XIV siècle (Ballestrieri, 1998). Dès cette époque, le Chianti apparaît comme un vin de référence. Le vignoble de Chianti est le premier espace viticole européen à avoir été mis en tourisme ${ }^{13}$. En effet, dès les années 1970, dans un contexte de déprise rurale, entrepreneurs étrangers et grandes familles terriennes décident de réhabiliter les bâtiments agricoles et de valoriser leur métier et leurs produits afin de développer le tourisme viticole dans leur région. Les formes d'accueil touristique sur l'exploitation se sont largement diffusées, accompagnées par une législation bien adaptée aux petites structures (Perrin, 2010). Des châteaux et des villas hérités de la Renaissance et de l'époque moderne sont encore visibles au cœur des espaces agricoles. Les châteaux de Verrazzano (San Casciano in Val di Pesa), de Meleto, de Brolio (Gaiole in Chianti) sont autant d'édifices construits par les grandes familles locales ayant marqué l'histoire du Chianti (Anger, 2011).

Le développement de l'œnotourisme dans le Chianti a été porté par différents profils d'acteurs publics et privés qui ont largement contribué au processus de renaissance rurale, de valorisation et de promotion du patrimoine viticole. Que ce soit dans le domaine viticole ou dans la transformation du bâti et des paysages ruraux, les professionnels du vin et du tourisme mais également les collectivités locales sont incités à s'engager dans ce processus, à travers la création d'une offre œnotouristique (hébergement, dégustation, restauration, vente directe de vin et de produits locaux, musées, etc.), l'organisation de manifestations ou des actions d'aménagement (routes des vins, aménagement des voies) (Perrin et Randelli, 2008). Des guides locaux et des amateurs de vin proposent également des circuits de randonnée pédestre et des itinéraires de vélotourisme. Beaucoup de ces professionnels travaillent en partenariat avec des tour-opérateurs et des agences de tourisme locales et étrangères. Le château de Verrazzano passe ainsi par des agences localisées dans les communes du Chianti pour la promotion et la distribution de ses prestations. Certains propriétaires disposant de grandes exploitations se sont même ouverts au tourisme d'affaires, accueillant des groupes en déplacement pour incentives ou dans le cadre de séminaires, à l'image de la Villa Talente à San Casciano (Ballestrieri, 2005). Des exploitations de petite taille 
proposent également des services d'agritourisme à la ferme, mais la qualité des prestations y est souvent inférieure et le cadre est moins grandiose. La clientèle y est moins aisée que dans les grands domaines, plutôt italienne, de composante scolaire, avec un accueil moins luxueux mais plus personnalisé ${ }^{14}$ (Perrin, 2010). Les deux catégories d'exploitation ne ciblent pas nécessairement la même clientèle, notamment s'agissant de l'hébergement. Tandis que les grandes propriétés sont positionnées sur le segment haut de gamme (200 euros en moyenne la chambre pour une nuit), les hébergements aménagés dans les plus petites exploitations se situent entre le milieu et le haut de gamme (100 euros la nuit en moyenne) (Anger, 2011).

Le terroir du Chianti Classico est aujourd'hui très dynamique, accueillant chaque année un peu plus d'un million de visiteurs, disposant d'un fort pouvoir d'achat. En 2018, le Chianti a ainsi attiré 1208307 visiteurs (touristes et excursionnistes). Ce tourisme est caractérisé par le poids important des étrangers parmi les visiteurs, qui représentent $62 \%$ des arrivées et $65 \%$ des nuitées. La plupart sont originaires d'Allemagne, des États-Unis, du Royaume-Uni et de la France (Observatoire régional du tourisme, 2019). En Italie, le produit œnotourisme correspond à près de 5 millions de touristes et un chiffre d'affaires estimé à 5 milliards d'euros. Les grandes fermes viticoles, elles-aussi, tirent bénéfice de la vente directe: l'analyse des 25 plus grandes caves italiennes, effectuée par Mediobanca en 2014, indique que 8,4\% de leur chiffre d'affaires provient du point de vente à la cave et que ce pourcentage monte à $16,6 \%$ pour les vins haut de gamme (Mediobanca, 2014). Les aspects positifs de l'œnotourisme ne se limitent pas aux fermes, d'autres activités peuvent exploiter les revenus injectés dans l'économie locale. Le rapport de l'Institut national de statistique italien évalue que, pour 10 euros dépensés à la cave, 50 euros l'ont été sur le reste du territoire. Les œnotouristes ne sont pas seulement attirés par les produits locaux, mais aussi par le territoire. Ils sont considérés parmi les catégories de clientèles effectuant les plus grandes dépenses journalières. Selon ce même rapport, pour chaque euro dépensé en vin, l'œnotouriste en dépense quatre pour des achats d'autres types. L'achat de bouteilles dans les caves représente seulement la moitié de ce qu'ils dépensent (Institut national de statistique, 2012).

18 L'œnotourisme et les routes viticoles sont basés sur des activités douces, pouvant ainsi générer des retombées économiques. Ils contribuent également à faire connaître une région viticole et agissent dès lors comme outils de promotion et de marketing territorial. Le succès du tourisme viticole dans le Chianti nous invite à nous interroger sur les potentialités de développement de l'œnotourisme dans la région du Cap Bon (Tunisie), mais aussi à tenter de comprendre l'émergence d'une fréquentation œnotouristique et à montrer dans quelles conditions la région pourrait intégrer la valorisation de son paysage et de son patrimoine viticole colonial dans les politiques de diversification du produit touristique tunisien. Ainsi, paysages, patrimoine viticole, produits du terroir issus de l'agriculture biologique, dégustés et visités au moyen de mobilités lentes (pédestre, équestre, à vélo) pourraient permettre une montée en gamme et fournir un complément au tourisme de masse centré sur les pratiques balnéaires. 


\section{L'œnotourisme dans la péninsule du Cap Bon : les pratiques d'une forme de tourisme alternatif}

\subsection{Histoire, paysage, architecture et patrimoine viticoles au Cap Bon : vecteurs de l'attractivité touristique d'un territoire rural}

La péninsule du Cap Bon, située au nord-est de la Tunisie, est depuis l'Antiquité le pays des vignobles. Les terres de cette région ont été occupées et exploitées par l'Homme depuis les périodes les plus reculées du passé. L'historien grec Diodore de Sicile décrit les paysages du Cap Bon à l'époque punique en tant qu'arrière-pays de Carthage, et rend compte de la richesse agraire et viticole de la région et du savoir-faire de ses habitants (Aounallah, 2002). Les premières plantations de vignes remontent à la civilisation carthaginoise, bien avant l'arrivée des Romains. Les Carthaginois furent les premiers, dans le monde antique, à faire des études scientifiques sur la viticulture et l'œnologie. La légende dit d'ailleurs que c'est la richesse du Cap Bon qui suscita la jalousie de Rome et attira sur Carthage la colère de l'Empire. L'agronome Magon, qui a vécu dans la Carthage phénicienne vers 140 av. J.-C., fut l'auteur du premier traité d'agronomie viticole en langue punique et consigne, dans son traité en 28 volumes, des pratiques qui sont toujours en usage de nos jours (Nasser, 1960). Des fouilles archéologiques réalisées par l'Institut national du patrimoine tunisien entre 1994 et 2002 dans le site de Henchir Dhouhek, à l'intérieur du terroir de Mornaguia (Takelsa), témoignent de cette longue tradition de viticulture. Les fouilles ont révélé la présence d'un complexe de production de vin, situé au milieu des vignobles, en excellent état de conservation. Le domaine remonte à la fin de l'Antiquité ( $v^{e}$ siècle) et comporte un pressoir à huile, un chai à vin, une aire de séchage des céréales, ainsi que des salles de stockage des produits du domaine (Ghalia, 2007). D'anciennes mosaïques romaines représentant des vignobles ou des scènes de dégustation du vin sont aujourd'hui exposées au musée national du Bardo à Tunis, qui possède l'une des plus belles collections de mosaïques romaines du monde (cf. illustration 1 ). L'arrivée de l'islam, dès le $\mathrm{VII}^{\mathrm{e}}$ siècle, fera presque disparaître la culture de la vigne dans le pays pendant plus de 1000 ans. Il faudra attendre 1881, avec l'instauration du protectorat français de Tunisie, pour voir la production du vin relancée. Durant la période coloniale, la viticulture tunisienne s'est beaucoup développée par l'extension des vignobles, la construction des caves et la création des appellations d'origine contrôlées (Poncet, 1962) (cf. illustration 2). 
Illustration 1. Mosaïque romaine du $\|^{\mathrm{e}}$ siècle après $\mathrm{J}$.-C., représentant une scène de dégustation du vin, découverte à Dougga (musée du Bardo)

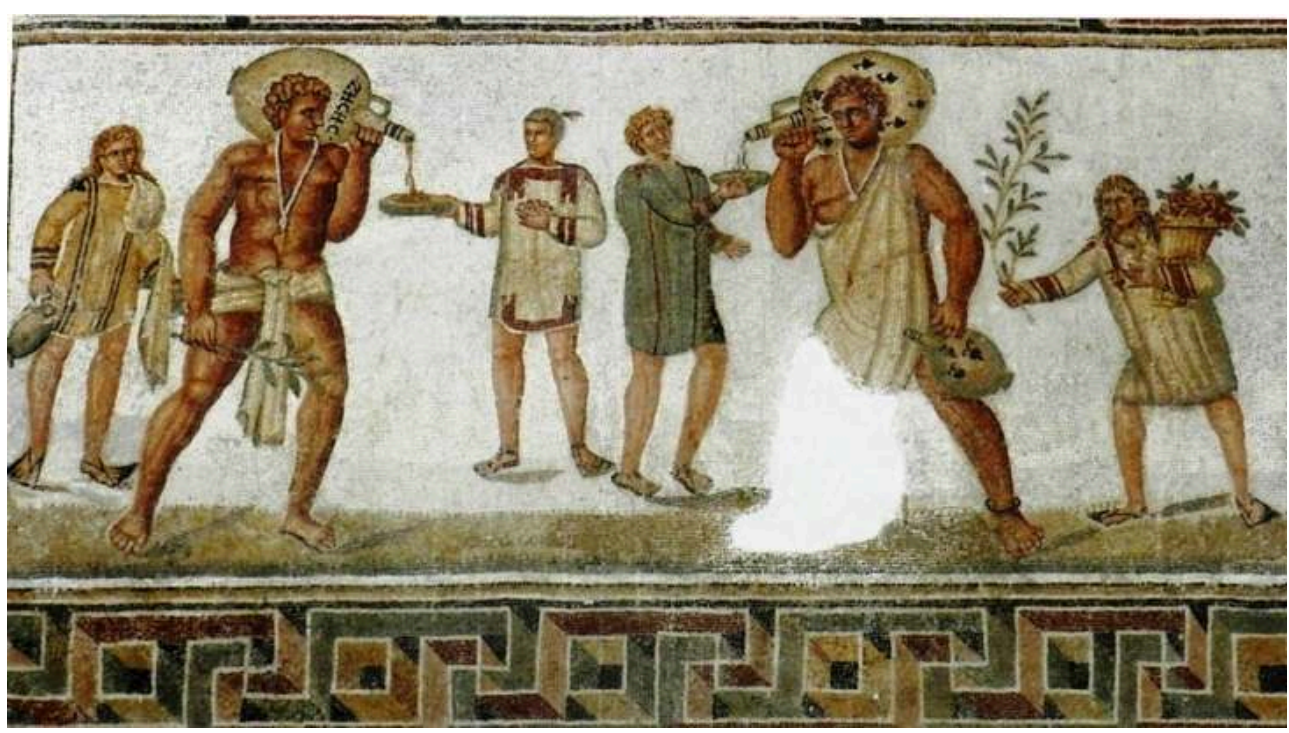

Photo : Mohamed Souissi.

Illustration 2. Dessin de désherbage d'un vignoble dans la plaine de Grombalia (période coloniale)

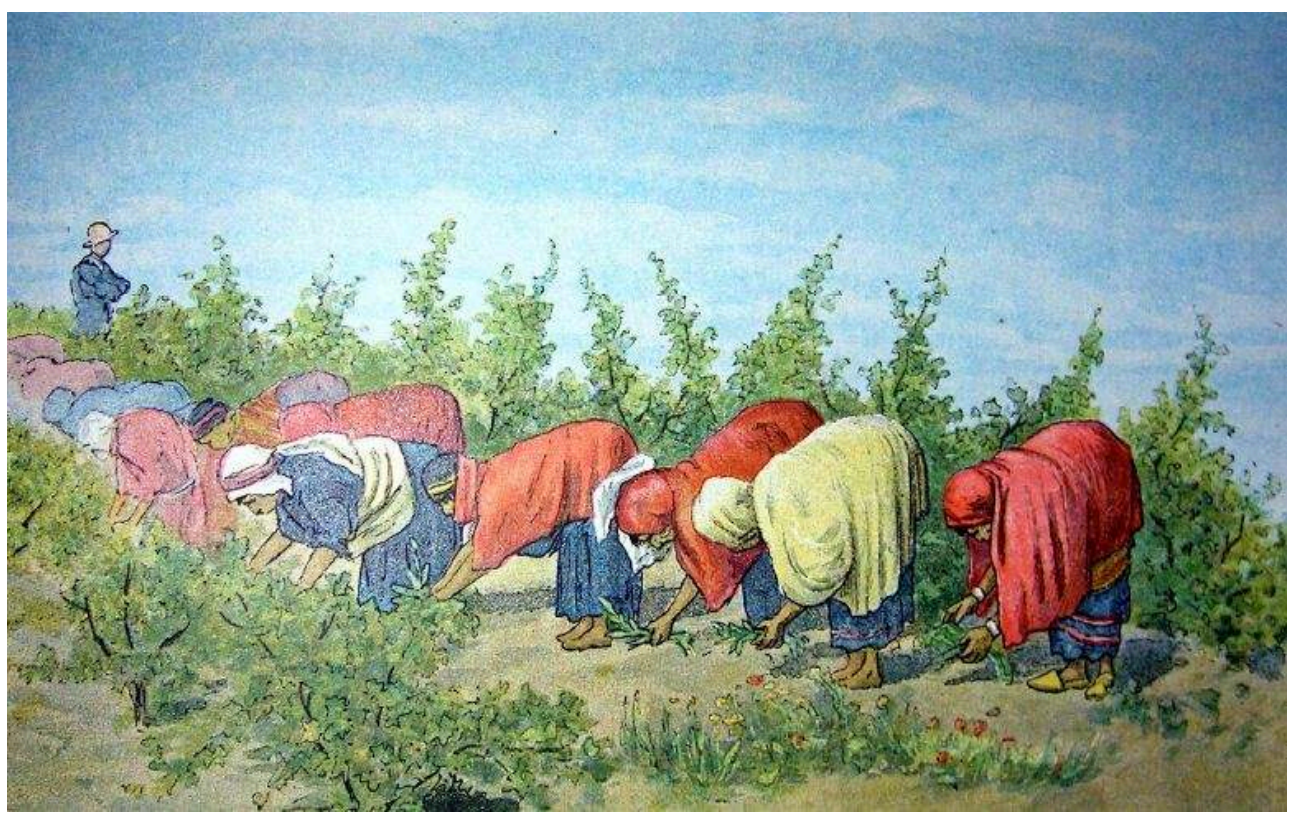

Photo : Charles Lallemand (La Tunisie, pays de protectorat français, Paris, 1892).

Les paysages ruraux et viticoles de la péninsule du Cap Bon sont généralement reconnus parmi les formes les plus remarquables de paysages résultant de l'activité agricole de la région, à la fois par la marque qu'ils impriment sur le pays et par les traditions culturelles qui leur sont associées. La côte orientale du Cap Bon est caractérisée par un paysage agricole diversifié. Les cultures dominantes sont les oliviers, l'arboriculture fruitière, les vignes et, surtout, le maraîchage ${ }^{15}$ (Sethom, 1977). Les territoires ruraux et viticoles de la plaine de Grombalia ${ }^{16}$ et les collines de Takelsa offrent un paysage viticole original sur la rive sud de la Méditerranée. Les terres plantées en vignes à raisin de cuve et en vignes à raisin de table (muscat d'Alexandrie et 
surtout muscat d'Italie) s'étendent à perte de vue. Dans leur composante viticole, les espaces ruraux du Cap Bon méridional constituent l'un des espaces les plus étendus de la péninsule. Les terroirs de Grombalia, Bouargoub, Soliman et Korba assurent $65 \%$ de la production nationale de vigne : de Fondouk-Jedid à Bouargoub, la viticulture s'étend sur près de 15000 hectares, soit $60 \%$ des vignobles du pays (Chabani, 2012). Un renouvellement des cépages et des caves vinicoles a pu se faire grâce à l'action des sociétés de mise en valeur vinicole et aux partenaires étrangers, en particulier italiens. Certains crus de Kélibia (chardonnay et muscat), des domaines de Neferis et de Saint Augustin à Khanguet Hojjej (blanc et rouge) commencent à se tailler une solide réputation à l'échelle internationale ${ }^{17}$.

La vigne était la culture la plus spéculative pour les colons français et italiens ${ }^{18}$. Elle profitait de prix favorables sur le marché français. Au total, la plaine de Grombalia et les collines de Takelsa regroupaient $91 \%$ des terres coloniales et $82 \%$ des agriculteurs européens de la péninsule du Cap Bon (Souissi, 2016). C'est la région de la Tunisie où la marque de la colonisation était et est encore la plus visible. Les colons agriculteurs ont construit dans ces territoires ruraux des bâtiments et des corps de fermes bien intégrés au paysage. Ces fermes jalonnent le paysage et se distinguent de loin grâce à leur architecture particulière, sous forme de petits palais particuliers, et aux toitures inclinées en tuiles rouges de leur cave (Brahim Kortass, 2006) (cf. illustration 3). Cette architecture viticole coloniale, composée de fermes, de chapelles, d'observatoires, de pressoirs à vin, est devenue au fil du temps une composante clé du paysage, permettant d'identifier le paysage agricole de la plaine de Grombalia et des collines de Takelsa. Les bâtisses de Bouargoub (Dar Mussolini), de Port Prince, de Mornaguia-Takelsa (pressoir de l'archevêché de Carthage) et de Khanguet el Hojjej (le château la Rosière, le château Saint-Augustin ${ }^{19}$ et les caves de Licari, Neferis, etc.) traduisent l'empreinte de cette période coloniale (Souissi et Rieucau, 2018) (cf. illustration 4).

Illustration 3. Architecture coloniale du patrimoine viticole : château du domaine Neferis

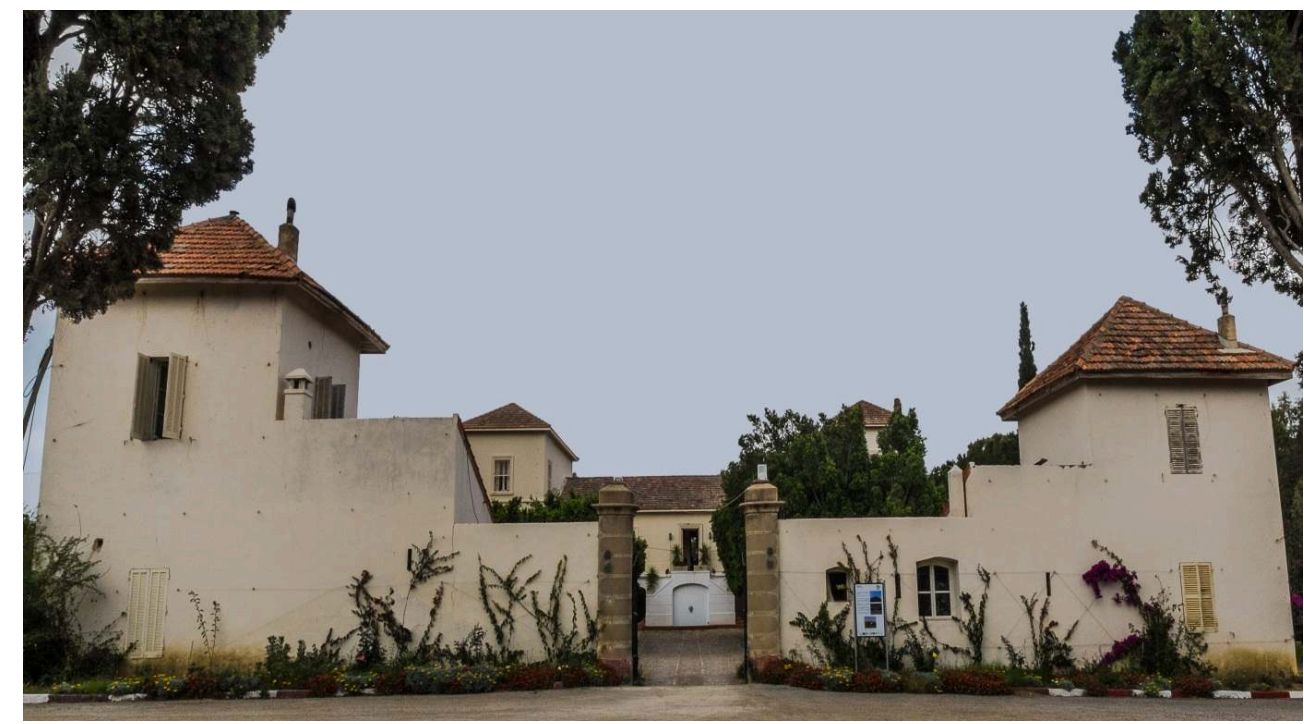

Photo : Mohamed Souissi. 


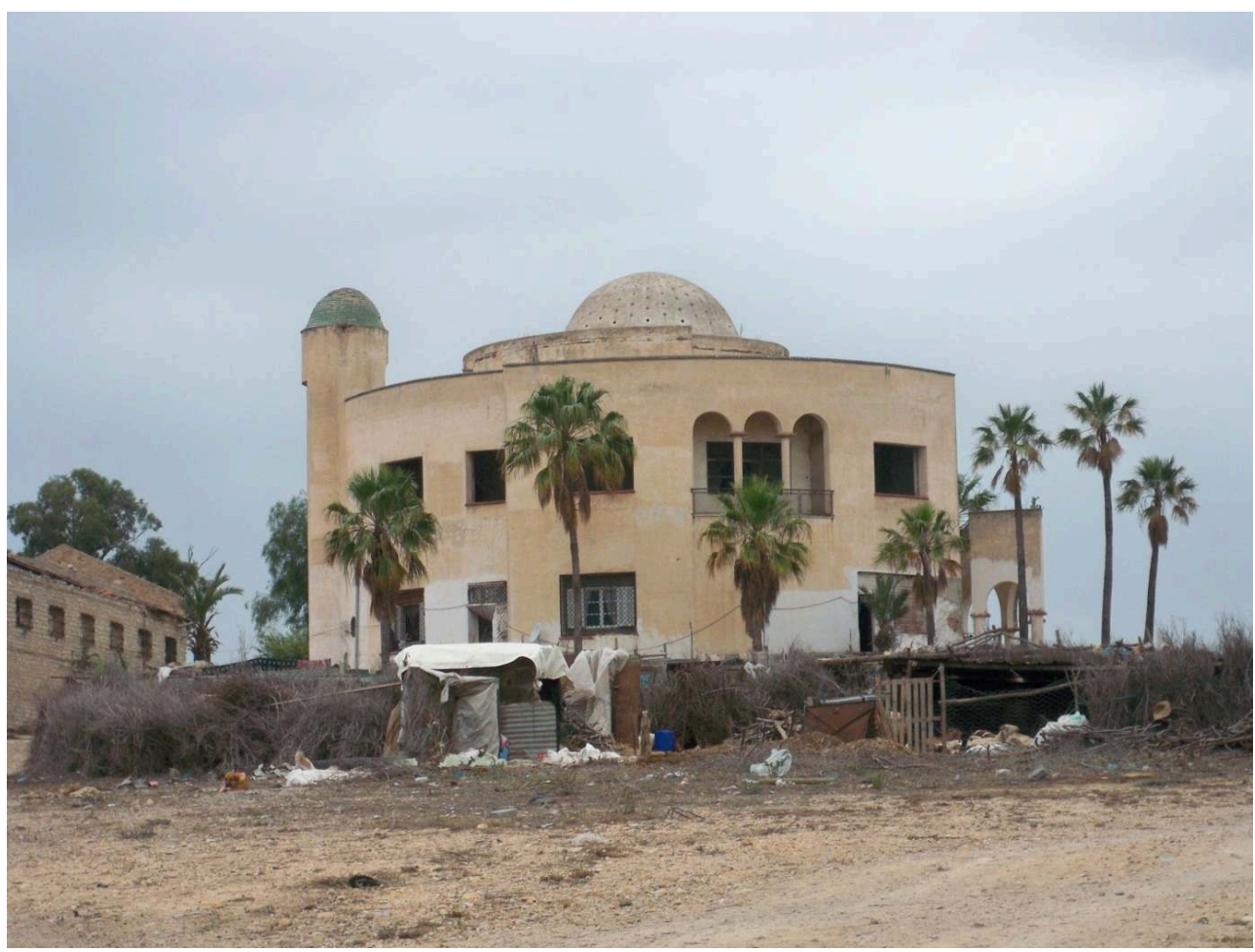

Photo : Mohamed Souissi

Le patrimoine viticole colonial de la Tunisie ${ }^{20}$ compte aujourd'hui plus de 1600 édifices, dont la majorité se concentre au Cap Bon. Ces édifices sont tous répertoriés en dehors des espaces urbains, et $60 \%$ de ces constructions sont des gîtes ruraux (930 gîtes). Ces constructions liées au vignoble symbolisent l'expression culturelle et historique du vin produit (Souissi, 2016). Ces monuments, menacés aujourd'hui de destruction et de disparition, surtout après la révolution de 2011, méritent l'attention, en vue d'une valorisation et d'une réaffectation en gîtes d'étapes dans le cadre de la mise en place d'un circuit à thème de sites éco-culturels dans les territoires ruraux. Certaines installations sont aujourd'hui désaffectées, comme les caves de Licari et le pressoir de l'archevêché de Carthage ( $c f$. illustration 5). D'autres fonctionnent encore, à l'instar de celles de l'Union centrale des coopératives viticoles à Bir Drassen ${ }^{21}$, de Septume et Atlas à Bouargoub et de celles de la Rosière à Khanguet El Hojjej. Ces caves et pressoirs sont des témoins de leur époque: elles présentent généralement une façade très bien architecturée où l'on trouve la gravure des noms des propriétaires des grandes familles ainsi que les dates de leur construction (Licarie : 1918, l'Archevêché : 1933, Neferis : 1928, etc.) (Brahim Kortass, 2006). Ces propriétaires ont également laissé une empreinte forte sur l'environnement quotidien de la population locale: on retrouve jusqu'à ce jour le nom de ces grandes familles sur les étiquettes des vins. D'autres domaines, à l'instar du château Neferis, intègrent une chapelle de province française; d'où la dimension culturelle conférée au vignoble par ce patrimoine religieux. 


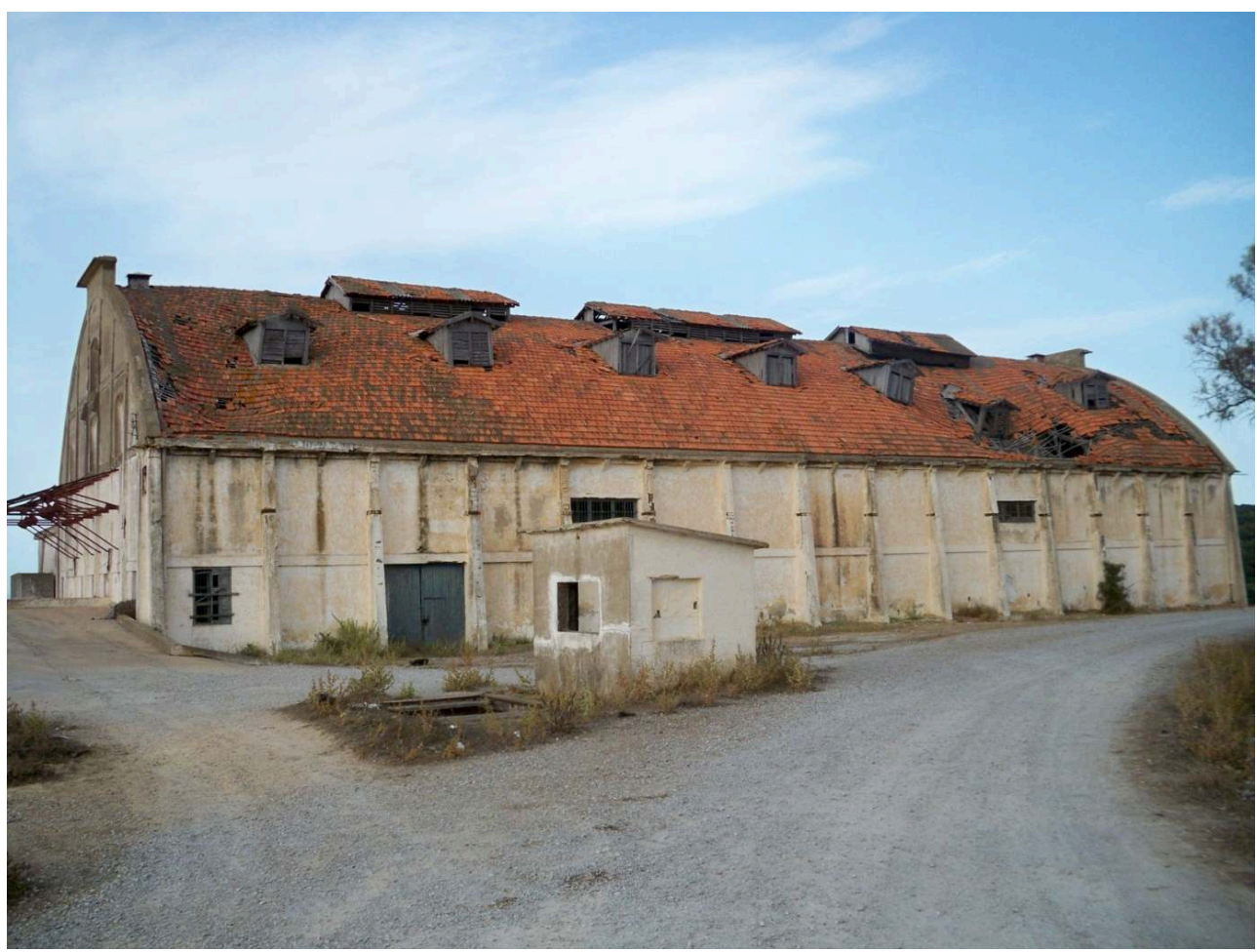

Photo : Mohamed Souissi.

Au-delà de ce potentiel touristique paysager et vitivinicole, les territoires ruraux des anciens villages de la péninsule du Cap Bon sont facilement accessibles depuis la capitale, Tunis, et le pôle touristique de Nabeul-Hammamet, grâce à la proximité de la route nationale N1 et de l'autoroute Tunis-Sfax. Enfin, ces territoires ruraux abritent également plusieurs vestiges puniques, romains, berbères et musulmans, encore visibles dans la région (Aounallah et Fantar, 2006). Parmi les vestiges les plus remarquables, on trouve les aqueducs romains à l'ouest de Grombalia et au sud du village Turki, ainsi que deux importants bourgs fondés par les Andalous expulsés d'Espagne au début du XVII ${ }^{e}$ siècle (vers 1609) ${ }^{22}$. Les monuments antiques de Ksar Majer à Sammech, de Chul-Medeina à Beni Khalled, de Tubernuc, de Vina-Henchir Maden et de Kourchine confèrent à ces territoires ruraux viticoles une dimension historique, ancrée dans la mémoire collective.

\subsection{L'ouverture des domaines viticoles au tourisme dans la péninsule du Cap Bon : fréquentation et circuits d'œnotourisme}

En l'absence d'une politique publique de développement du tourisme dans l'espace rural, il a fallu attendre les années 2000 pour que les exploitations viticoles commencent à s'ouvrir au tourisme dans la péninsule du Cap Bon, à travers notamment l'aménagement d'espaces et de petites structures destinés à l'agritourisme au sein des fermes viticoles. L'ouverture des caves au public est le fait d'une initiative privée : des vignerons indépendants, passionnés, de sociétés viticoles à la recherche d'une opération de marketing afin de renforcer leur image nationale et internationale, ainsi que leurs marchés en général. Il s'agit d'initiatives d'œnotourisme que l'on qualifie 
localement d'agritourisme, où les agriculteurs apprécient l'échange social avec les visiteurs de la ferme. Ces acteurs particuliers fonctionnent individuellement et proposent un accueil au public sur leur propriété afin de faire découvrir aux visiteurs leurs produits mais aussi leur histoire et leur terroir. Ils se décrivent avant tout comme des exploitants agricoles, soucieux de la qualité et de la commercialisation de leur vin. Bien que l'objectif soit avant tout économique, il n'en demeure pas moins que les ambitions sont aussi celles de la protection, de la conservation et de la valorisation. Dans le cadre des entretiens réalisés, les propriétaires des domaines viticoles considèrent le petit patrimoine viticole à l'image de l'ensemble des traces patrimoniales du Cap Bon et pensent que ce sont ces petits aspects qui devraient être protégés pour leur rareté et leur signification. C'est une des raisons pour lesquelles ils ouvrent leur cave, afin de partager un objet patrimonial qu'ils ont la chance de posséder.

Certains propriétaires, à l'instar de ceux des domaines Neferis, Atlas ou Bir Drassen, organisent des dégustations et des visites guidées de la propriété, pratiquent la vente directe et offrent des services de restauration ( $c f$. illustration 6). Ces propriétaires ont aménagé dans leurs domaines un chai où s'effectuent les dégustations et les ventes de produits. C'est généralement là que les propriétaires parlent de leurs vins, mais également de l'histoire du Cap Bon et de toutes les spécificités de la région; c'est en ce sens qu'ils font de la valorisation ( $c f$. illustration 7). En tant que passionnés de leur domaine familial, ils se sont largement documentés et partagent avec plaisir les informations ou documents qu'ils ont pu acquérir. Ils offrent ainsi aux visiteurs la possibilité d'entrer en contact avec une histoire riche, qui a su laisser perdurer des traces du patrimoine colonial viticole, que certains n'omettent pas de mentionner. Ainsi, ouverts au tourisme viticole, ces domaines exercent des activités d'agritourisme, en partenariat avec des associations de randonnée, des consulats de pays étrangers en Tunisie et des clubs privés de divers secteurs professionnels (médecins, ingénieurs, enseignants, etc.). La promotion des activités des caves viticoles en Tunisie s'effectue essentiellement par le bouche-à-oreille, sur les sites internet et les réseaux sociaux, avec la collaboration des magazines spécialisés, mais également à travers la participation aux différents concours et salons internationaux des vins et de la gastronomie (cf. illustration 8). 
Illustration 6. Aménagement d'un espace de restauration (domaine Atlas)

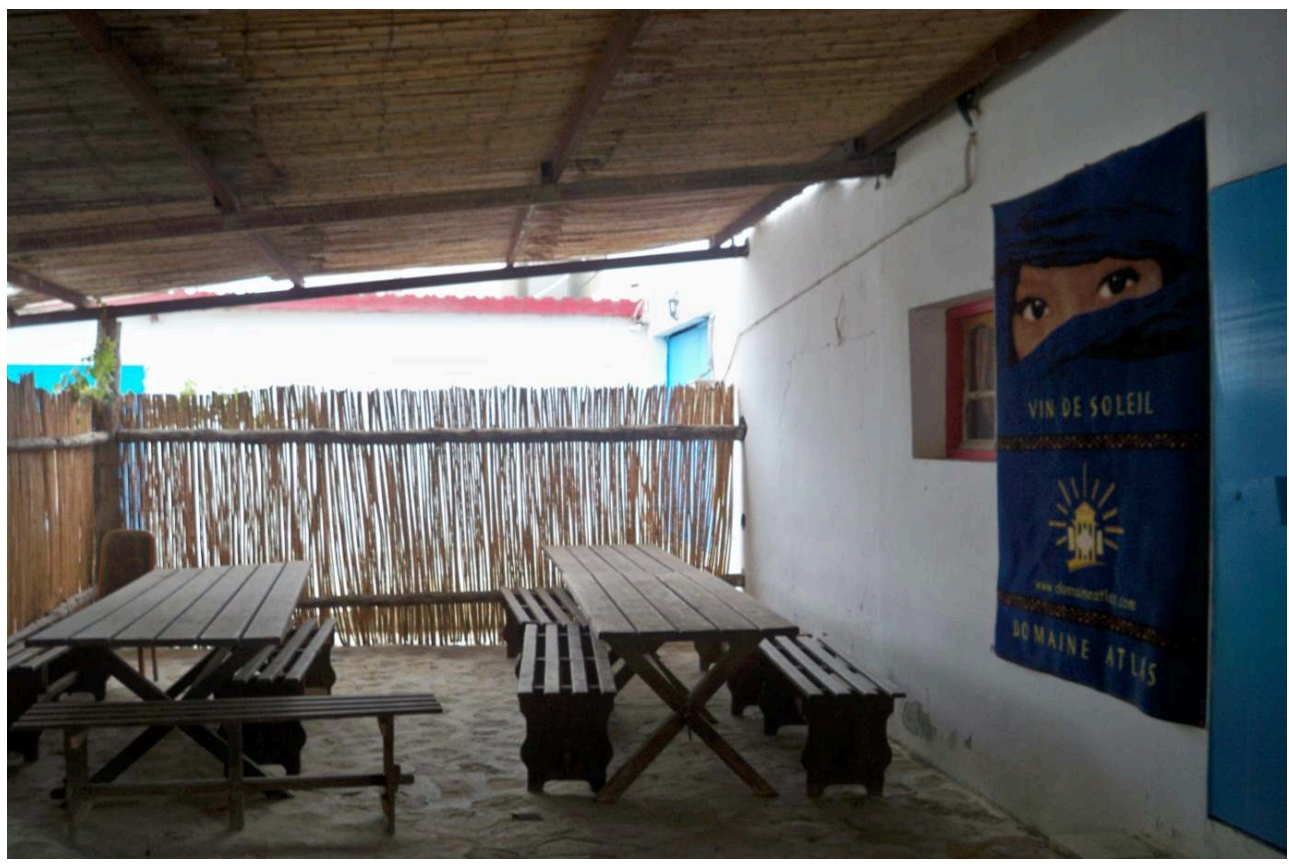

Photo : Mohamed Souissi.

Illustration 7. Visite guidée et dégustation de vin (cave de Bir Drassen)

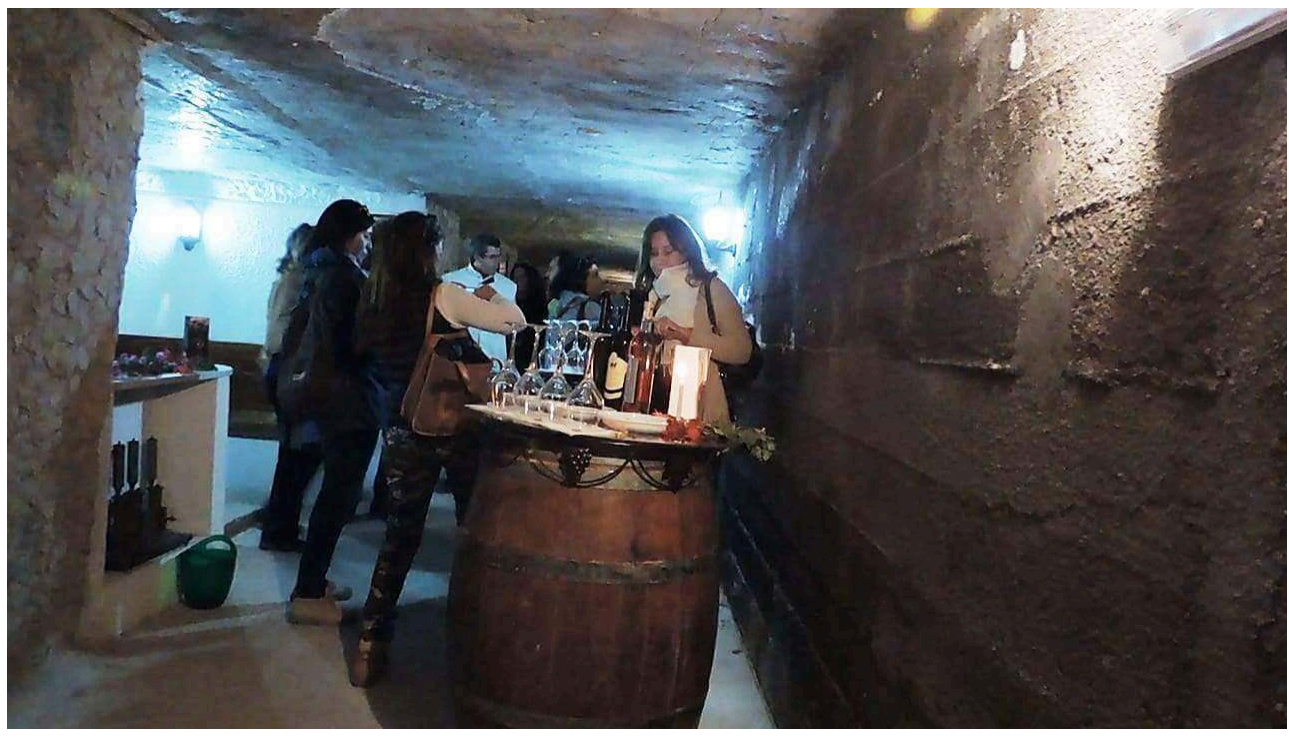

Photo : Gérant de la cave de Bir Drassen. 
Illustration 8. Exemple de prospectus publicitaire diffusé sur Internet (cave Magon Majus)

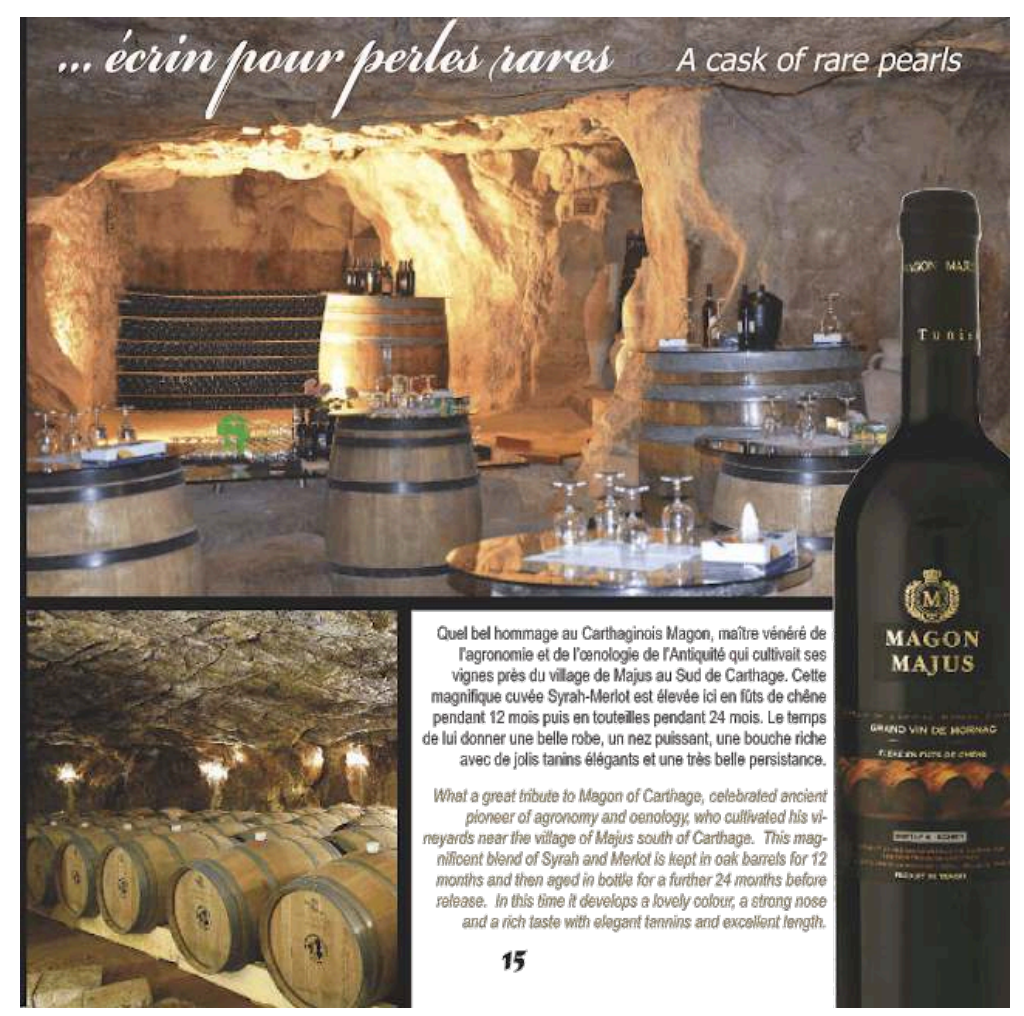

Photo : Les vignerons de Carthage (http://oenotourisme-tunisie.blogspot.com).

Il est vrai que le flux touristique engendré actuellement par cette activité reste encore très modeste, mais sa fréquentation est en émergence comme l'ont montré nos entretiens avec des acteurs locaux ${ }^{23}$. Des entretiens ont, d'une part, été menés auprès de caves coopératives et d'entreprises vinicoles dans la région du Cap Bon; ils ont été complétés, en amont et en aval, par des enquêtes réalisées au moyen de questionnaires auprès des randonneurs et des visiteurs des domaines viticoles. L'enquête par questionnaires conduite dans les fermes viticoles Atlas et Bir Drassen, en mars et en avril 2016, nous a permis de faire apparaître que les Tunisiens représentent $83 \%$ des personnes interrogées, la part des étrangers ne dépassant pas $17 \%$ du total des visiteurs. Ces visiteurs appartiennent à des catégories socioprofessionnelles supérieures et fréquentent le domaine principalement en couple ou en famille. Le choix de ces vignobles est motivé, pour une majorité d'entre eux, par le cadre et les paysages qu'offrent les espaces ruraux de la région du Cap Bon (56\%). L'œnogastronomie est le deuxième motif de fréquentation mentionné $(23 \%)$. En troisième position sont énoncées l'hospitalité et la chaleur humaine, qui permettent aux visiteurs de renouer avec des habitudes et des traditions locales qui participent de l'esprit de groupe et de rassemblement ( $21 \%)$. Ainsi, d'après les résultats de nos enquêtes, le terroir viticole de cette partie méridionale du Cap Bon constitue une ressource attractive pour une population tunisienne et étrangère relativement aisée.

Parallèlement, des guides locaux et des amateurs de vin, des associations de randonnée pédestre, des clubs de loisirs, des agences de voyages ${ }^{24}$ proposent des circuits pédestres et des itinéraires de vélotourisme qui relient les différents vignobles et caves situés dans la péninsule du Cap Bon. Ces acteurs commercialisent des circuits, des routes des vins qui permettent de parcourir le patrimoine viticole (caves) édifié par les colons 
français et italiens au début du $\mathrm{xx}^{\mathrm{e}}$ siècle. Le patrimoine bâti de ces grands domaines (Licari, Michaud, Saint Joseph Hollande, Calatrasi) fonde la ressource touristique de cet œnotourisme. Le discours promotionnel de ces acteurs affiche la destination du Cap Bon dans son contexte historique et culturel, mais également dans ses paysages et ses productions viticoles («Sur la route du Cap Bon », « Découvrir les traditions ancestrales du vin », « Rallye des caves de Tunisie », «Les origines du vin », "Grombalia, capitale de la vigne », etc.). Les circuits d'œnotourisme font référence au célèbre agronome carthaginois Magon, qui a tracé la route des vins et qui est aux origines de la culture de la vigne en Tunisie ${ }^{25}$. Ces circuits se déroulent sur 4 à 5 jours. Ils sont ponctués d'arrêts dans des sites archéologiques et historiques (Kerkouane, Néapolis, Port Prince, Tubernuc, Mornaguia). Les itinéraires prévoient également des haltes viticoles (halte gastronomique: vins, produits du terroir), fondées sur des produits biologiques de terroir, associées à des dégustations de vin et à des visites de caves assurées par des guides locaux et des maîtres œnologues (Souissi et Rieucau, 2018). Cependant, nos entretiens ont révélé que les propriétaires de domaines viticoles refusent généralement de collaborer avec les hôteliers et les agences de voyages de la région en raison notamment du faible pouvoir d'achat des touristes acheminés par ces derniers mais également dans le but de conserver le caractère authentique et familial du lieu. Cette difficile implication des propriétaires interroge quant à l'avenir du développement de l'œnotourisme dans les vignobles du Cap Bon.

\section{L'avenir du développement de l'œnotourisme dans les vignobles du Cap Bon : les enjeux de mise en tourisme du patrimoine viticole}

\subsection{Les limites du développement de l'œnotourisme : une faible implication des acteurs}

Malgré l'engagement des acteurs locaux pour développer les activités du tourisme viticole dans les vignobles du Cap Bon, la fréquentation des territoires et des domaines viticoles reste faible. L'œnotourisme ne représente pas encore une réelle offre alternative ou, du moins, sa promotion n'est pas ou peu visible. Plusieurs éléments permettent d'expliquer pourquoi l'œnotourisme se heurte à des contraintes et prend autant de temps à s'implanter et à se développer. Tout d'abord, la situation n'est pas simple pour les vignerons et les agriculteurs qui pourraient être concernés par l'accueil chez eux de visiteurs et leurs hésitations sont compréhensibles. Ils n'y voient pas d'intérêt tant qu'ils n'ont pas besoin de revenus complémentaires. Selon nos entretiens, menés auprès de caves coopératives et d'entreprises viticoles situées dans la plaine de Grombalia, la période d'accueil des visiteurs coïncide avec le moment où le travail est le plus intense sur l'exploitation (Souissi, 2016). Les investissements nécessaires aux travaux d'entretien et de rénovation du patrimoine viticole peuvent également les démotiver, les vignerons tunisiens étant déjà très endettés.

Le désintérêt des instances dirigeantes (politiques, administratives, professionnelles) et l'absence de soutien financier aux porteurs de projets de restauration et de valorisation $\mathrm{du}$ patrimoine viticole (propriétaires de domaines familiaux, vignerons, sociétés viticoles) freinent le développement de cette forme particulière du tourisme. Cela se traduit par l'absence de panneaux et de cartes routières indiquant aux visiteurs la 
localisation des producteurs des domaines de vignobles, ainsi que le manque de documentation et d'information sur les potentialités du tourisme viticole dans la péninsule du Cap Bon. Les porteurs de projets manquent notamment de moyens humains, techniques et financiers pour un développement conséquent. Or, les besoins en développement de l'œnotourisme sont relativement importants, car le tourisme dans les territoires ruraux suppose l'acquisition de compétences nouvelles (accueil, montage de projet...) et touche des domaines nouveaux et coûteux (promotion, techniques de commercialisation...). Il a aussi besoin de fonds publics, qui lui font actuellement défaut, alors que les lourds investissements qu'il nécessite ne peuvent être pris en charge par les seuls vignerons et acteurs privés.

30 Au-delà des contraintes financières et logistiques, c'est le vide juridique dans lequel évolue le tourisme dans les territoires ruraux qui pose problème. La réglementation du tourisme ne prend en considération que les activités se déroulant dans les zones définies comme touristiques ou sur les trajets d'hôtel à hôtel et les routes principales, voire vers des sites touristiques bien précis. L'absence d'une loi qui réglemente les circuits et les routes des vins et ayant pour objet de protéger et de valoriser les territoires viticoles ainsi que leurs produits ne favorise pas le développement de l'œnotourisme. Si l'œnotourisme et la valorisation du patrimoine qui lui est lié ne font l'objet d'aucune réglementation spécifique, l'encadrement réglementaire du secteur touristique en Tunisie apparaît par contre, dans les entretiens, comme une contrainte pour certains acteurs et contribue à décourager les agriculteurs et les gérants de caves coopératives et d'entreprises viticoles. Les lois sur l'agriculture, celles sur l'hébergement et l'hôtellerie, la restauration et le commerce de boissons alcooliques, ainsi que les autorisations souvent nécessaires pour les circuits et les randonnées dans l'espace rural ne facilitent pas toujours le développement de ce nouveau tourisme. Dans la plupart des cas, les services de restauration, de dégustation et d'hébergement proposés par certaines fermes viticoles au Cap Bon sont pratiqués en toute illégalité, c'est-à-dire sans être déclarés. À cela s'ajoutent d'autres contraintes législatives qui se posent aujourd'hui en matière de construction, très défavorables à la création de logements nouveaux sur un domaine agricole.

31 En outre, la communication n'est pas toujours évidente entre les différents intervenants et acteurs locaux (vignerons indépendants, caves coopératives, tissu associatif, communauté locale, guides locaux, investisseurs passionnés par le vin et les vignobles, etc.). Cette coordination difficile est principalement liée à l'absence d'une organisation et d'une administration des activités touristiques dans les territoires ruraux. En effet, les acteurs et les structures impliqués dans le tourisme viticole sont, pour la plupart, récents et peu connus. Ils assurent, souvent indépendamment les uns des autres, le développement et la promotion des prestations œnotouristiques. De même, les différents services de l'office du tourisme tunisien (direction des études, direction des nouveaux produits, direction marketing, etc.) sont encore noyautés par l'hôtellerie balnéaire. Aujourd'hui encore, le tourisme rural ${ }^{26}$ est, pour beaucoup d'acteurs, une branche marginale ou mineure du tourisme en général et est souvent dénigré par rapport au tourisme conventionnel.

32 Enfin, au-delà de ce cadre administratif, se pose aujourd'hui la question de la représentation sociale du tourisme en Tunisie, qui reste très dépendante du modèle conventionnel centré sur l'activité balnéaire. Malgré l'intérêt de quelques catégories sociales pour l'agritourisme, notamment les cadres supérieurs, les entretiens menés 
auprès de la population locale ont montré que, pour la majorité des ruraux, le tourisme s'associe à la mer, aux hôtels et appartements balnéaires construits sur la côte pour l'accueil des masses. Développer l'œnotourisme alors que domine cette conception suppose un changement de mentalités qui n'associe pas le champ et l'activité agricoles à des images négatives de pénurie et de difficulté économique.

Dans l'ensemble, même si l'émergence d'une fréquentation viticole a été démontrée ces dernières années dans la péninsule du Cap Bon, l'offre œnotouristique est très limitée, pour différentes raisons plus ou moins fondées. Cette situation d'incertitude ne se prête pas aux investissements coûteux nécessaires. Elle n'autorise pas non plus une large communication auprès des vignerons et des agriculteurs, mais également auprès du grand public et des partenaires touristiques nationaux ou étrangers. Cette situation n'est pourtant pas définitive et va peut-être être amenée à évoluer dans un avenir proche. Les ministères du Tourisme et de l'Agriculture ont convenu de mettre en œuvre, prochainement, un accord concernant la mise en place d'un cadre réglementaire régissant l'exploitation des sites agricoles à des fins touristiques, tout en préservant leur vocation originale. Cela impliquera peut-être une évolution de la situation touristique en milieu rural et, plus particulièrement, dans les territoires viticoles.

\subsection{Les perspectives de l'œnotourisme : quel avenir pour les terroirs viticoles du Cap Bon?}

La mise en tourisme du patrimoine viticole dans la péninsule du Cap Bon exige une diversité d'actions de la part des acteurs locaux et des instances dirigeantes (politiques et administratives). L'administration du tourisme, les communes et les professionnels des domaines viticoles doivent mobiliser leurs efforts et leurs moyens (législatifs, financiers, techniques, logistiques, etc.) pour le développement de la filière œnotouristique au Cap Bon. Le regroupement des acteurs permet de faciliter certaines étapes, comme la publicité, et de garantir une certaine qualité. Ainsi, la population des aires urbaines et les stations touristiques balnéaires en crise ont la possibilité de profiter de la proximité de ces territoires ruraux pour relancer l'économie du tourisme et développer d'autres formes de tourisme alternatives et complémentaires. Le tourisme viticole permet d'allonger la saison touristique estivale en renforçant les « ailes de saison » (avant-saison et arrière-saison : avril, mai, juin, septembre, octobre). Aussi, les acteurs impliqués dans l'œnotourisme pourraient procéder à la création d'un système de labels nationaux reconnaissant la spécificité d'un savoir-faire et d'une zone de production viticole dans la péninsule du Cap Bon. Tout comme la communication sur l'histoire de la viticulture, la beauté des paysages et le caractère traditionnel de la culture locale, les labels et les appellations participent à la diffusion d'une image de qualité du vin et au discours pouvant amener les consommateurs à porter un regard positif sur les produits locaux et la région productrice.

En collaboration avec les communautés locales, les propriétaires des domaines viticoles et les intervenants du secteur touristique pourraient attribuer une valeur patrimoniale aux activités et aux paysages et adopter des pratiques de conservation (entretien, réhabilitation). Sur place, le vigneron est appelé à mettre en scène son savoir-faire et l'art de vivre de sa région pour répondre aux attentes des visiteurs désireux de découvrir et d'apprendre les traditions locales. Aussi, au niveau de l'accueil et de 
l'accessibilité, les acteurs publics sont appelés à équiper les territoires viticoles en panneaux de signalisation et en structures d'accueil, et à veiller à l'entretien des infrastructures de transport. Cette mobilisation des acteurs pourrait être accompagnée par un projet de loi concernant les routes des vins. Ce projet de loi pourrait porter, entre autres, sur la signalétique (les panneaux routiers) dans le but de favoriser la reconnaissance du très large réseau de paysages viticoles, de caves, de châteaux et de fermes qui structurent les routes viticoles. Il pourrait contribuer à réglementer le secteur et à faire des routes du vin un instrument d'organisation, de gestion et de promotion du territoire dans chaque région de la péninsule du Cap Bon. En Italie, dans le terroir du Chianti Classico, la gestion des routes des vins est assurée par des structures au sein desquelles acteurs publics et privés collaborent. Il s'agit, le plus souvent, d'associations composées d'élus locaux, de producteurs et de restaurateurs qui œuvrent à l'harmonisation des panneaux indiquant aux visiteurs les caves, les musées, les boutiques de vin et les restaurants adhérents à la route du vin concernée.

En somme, l'État tunisien est amené à définir une stratégie de valorisation et de protection du patrimoine viticole colonial dans la péninsule du Cap Bon (bâti agricole, bâtiments d'habitation et bâtiments liés à l'exploitation), patrimoine qui est aujourd'hui menacé de disparition, souvent abandonné à son sort et n'a parfois plus d'usage agricole. Cette valorisation touristique passe par une phase de repérage et de connaissance, et nécessite une volonté de l'administration du tourisme, du ministère de l'Agriculture, des élus locaux, mais aussi des habitants et des associations impliquées dans ce domaine. L'État et les collectivités locales ont la possibilité d'intervenir par des mesures à caractère fiscal ou financier. Quant aux nombreux particuliers, ils pourraient intervenir pour sauvegarder, faire connaître ou valoriser des bâtiments agricoles. Ce travail difficile suppose que soit réalisé un véritable inventaire et que soit institué un suivi du devenir des bâtiments agricoles ainsi recensés. Enfin, l'avenir de l'œnotourisme au Cap Bon nécessite l'ajustement de l'offre aux exigences locales, en donnant plus d'importance à l'initiative privée et aux modèles de gestion et de promotion associatifs, dont le succès a déjà été démontré dans d'autres pays d'Europe, notamment en Italie et en France. Quant à la demande, il est nécessaire de choisir le segment qui convient au niveau local, en réalisant les études adéquates, étant donné la grande méconnaissance que l'on en a. Cela suppose de planifier un modèle de développement du tourisme viticole à l'échelle locale qui privilégie l'aspect territorial avec une approche trans-sectorielle par rapport à un développement de type fonctionnel/sectoriel.

\section{Conclusion}

Nos recherches approfondies sur la mise en tourisme du patrimoine viticole dans la péninsule du Cap Bon en Tunisie montrent que, malgré l'importance des aménités touristiques viticoles (qualité du paysage, richesse de l'architecture viticole coloniale), l'œnotourisme ne représente pas encore une réelle offre touristique alternative. Ce nouveau produit touristique demeure encore inorganisé, familial, associatif, dépourvu d'encadrement législatif et privé des processus de labellisation, pas encore à l'œuvre dans le pays. Il est difficile d'appréhender avec précision dans quelle situation se trouve actuellement l'œnotourisme et quel sera son avenir, que ce soit au niveau des produits, de l'hébergement, des circuits, des services de restauration et de dégustation ou dans sa 
globalité. Il est vrai que le flux touristique engendré par cette activité reste encore très modeste, mais il a été prouvé par des entretiens avec les acteurs locaux que ce nouveau produit de tourisme alternatif pourrait participer à l'avenir au développement économique des régions concernées, pour autant qu'on lui en laisse l'opportunité. La valorisation du patrimoine et des paysages viticoles, la préservation et la valorisation de la nature sont des atouts sur lesquels devrait s'appuyer l'œnotourisme en Tunisie face à la montée des préoccupations environnementales dans nos sociétés modernes. Le patrimoine naturel, culturel et historique des vignobles du Cap Bon constitue un élément essentiel de différenciation dans cette nouvelle forme de tourisme diffus, par opposition à un tourisme concentré fondé sur des stations balnéaires, tel qu'il s'observe le long du littoral tunisien. La patrimonialisation et le développement du tourisme viticole sont ainsi à mettre en relation avec la construction $\mathrm{du}$ « terroir rural Cap Bon ». Ce terroir correspond à un espace géographique et culturel unique. Il pourrait être composé de lieux relevant du patrimoine bâti et d'espaces agricoles, habités et exploités par une population rurale particulière, et dotés d'une culture propre construite autour du vin. Le terroir, une fois défini et reconnu par un système d'appellation et grâce à un système de communication étudié, poserait ainsi les bases de ce qui pourrait attirer les futurs touristes, dotés d'un fort pouvoir d'achat.

Enfin, nos enquêtes de terrain dans les territoires ruraux du Cap Bon ont montré que les petits patrimoines viticoles hérités de la période coloniale apparaissent largement sous-valorisés. La durabilité de ce patrimoine, menacé en grande partie de disparition de nos jours, est un élément essentiel de la durabilité du tourisme viticole dans la péninsule du Cap Bon. Sa valorisation doit être une volonté de l'ensemble des acteurs de l'espace rural (agriculteurs, population locale, associations, services régionaux du ministère du Tourisme et de celui de l'Agriculture). Ces acteurs sont appelés à se coordonner et à travailler en réseau dans le cadre de circuits ruraux et viticoles. La création d'une association de la route du vin, qui regrouperait les villages et les communes traversés par le circuit du vin, pourrait amener à dépasser les logiques de gestion institutionnelles traditionnelles et permettre de mobiliser les acteurs privés à l'échelle de leur territoire. Ainsi, non seulement l'offre œnotouristique serait développée et structurée, mais elle ferait l'objet, dans le futur, d'un suivi renforcé et de projets.

\section{BIBLIOGRAPHIE}

Nasser ABOU, Histoire de l'agriculture ancienne, Beyrouth, 1960.

Virginie ANGER, La mise en tourisme du patrimoine viticole : l'exemple du Chianti, master professionnel en tourisme, Université de Paris 1 Panthéon-Sorbonne, Institut de recherche et d'études supérieures du tourisme, 2011.

Samir AOUNALLAH, Le Cap Bon, jardin de Carthage. Recherches d'épigraphe et d'histoire romano-africaines (146 a.C-235 p.C.), Ausonius, 2002. 
Samir AOUNALLAH et Mounir FANTAR, À la découverte du Cap Bon : guide historique et archéologique, Tunis, Agence de mise en valeur du patrimoine et de promotion culturelle, 2006.

Giovanni BALLESTRIERI, Il turismo rurale nello sviluppo territoriale integrato della Toscana, Florence, IRPET, Regione Toscana, 2005 [https://www.irpet.it/storage/pubblicazioneallegato/ 49_Balestrieri.pdf].

Giovanni BALLESTRIERI, Agriturismo in Toscana, monitoraggio di un campione di aziende turistiche per la validazione della proposta per la classificazione delle attività agrituristiche della Regione Toscana, ARSIA, 1998.

Gérard BEAUDET, « Les routes touristiques à thème : entre marketing territorial et valorisation identitaire », Téoros, vol. 22, n² 2, 2003 [http://journals.openedition.org/teoros/1747].

Jacinthe BESSIÈRE, «Valorisation du patrimoine gastronomique et dynamiques de développement territorial. Le haut plateau de l'Aubrac, le pays de Roquefort et le Périgord noir ", Ruralia, $\mathrm{n}^{\circ}$ 6, 2000 [http://journals.openedition.org/ruralia/154].

Cheïma BRAHIM KORTASS, Les paysages viticoles des fermes coloniales de la plaine de Grombalia, diplôme de mastère paysage, territoire et patrimoine, Sousse (Tunisie), Institut supérieur agronomique de Chott-Mariem, 2006.

Bruno CARLIER, « Construire et dynamiser une route touristique », Pratiques économiques : initiatives et activités, 2000.

CENTRE D'ÉTUDES SUR L'INVESTISSEMENT SOCIAL, Ottavo rapporto sulla comunicazione. I media tra crisi $e$ metamorfosi, Milan, Franco Angeli Edizioni, 2009.

Abdelhamid CHABANI, Les transformations structurelles et spatiales des vignobles en Tunisie et ses conséquences géographiques, thèse de doctorat en géographie, Université de la Manouba à Tunis (texte en langue arabe), 2012.

Valérie DELIGNIÈRES, «L'authenticité des territoires ruraux touristiques en question. Réflexions au sujet de l'Auxois et du Morvan ", Revue géographique de l'Est, tome 38, n³, 1998 [https://doi.org/ 10.3406/rgest.1998.2340].

Guy DI MEO, Géographie sociale et territoires, Nathan, 1998.

Paul DUBRULE, L'œnotourisme : une valorisation des produits et du patrimoine vitivinicoles, rapport remis aux Ministres du Tourisme et de l'Agriculture », 2007 [https://www.vie-publique.fr/rapport/ 37896-loenotourisme-valorisation-des-produits-et-du-patrimoine-vitivinicoles].

Agnès DURRANDE-MOREAU, François H. courVoISIER et Anne Marie BOCQUET, « Le nouvel agritourisme intégré, une tendance du tourisme durable ", Téoros, vol. 36, n 1, 2017 [http:// journals.openedition.org/teoros/3009].

Taher GHAliA, Le Cap Bon El Watan El Qibli : le Pays, l'Histoire, les Hommes, Tunis, Centre de publication universitaire, 2007.

INSTITUT NATIONAL DE STATISTIQUE, Il primo conto satellite del turismo per l'Italia, statistiche report, Rome, Institut national de statistique, 2012.

Gilbert JACUETON, Le peuplement de la Tunisie, Paris, Motteroz, chambre des députés, $n^{\circ}$ 611, 1902.

Olivier LAZZAROTTI, « Tourisme et patrimoine : ad augusta per angustia / Tourism and heritage: ad augusta per angustia », Annales de géographie, tome 112, n 629, 2003 [https://doi.org/10.3406/ geo.2003.893].

Olivier LAzZARotTI, Patrimoine et tourisme. Histoires, lieux, acteurs, enjeux, Belin, 2011. 
Marie LEMARIÉ-BOUTRY, « Foncier viticole, patrimoines et œnotourisme : un modèle des prix hédoniques appliqué au vignoble bordelais ", Revue d'économie régionale et urbaine, $\mathrm{n}^{\circ}$ 4, 2016 [https://doi.org/10.3917/reru.164.0877].

Sophie LIGNON-DARMAILLAC, « Les routes des vins vers l'œno-gastronomie », Territoires du vin, 2019 [http://preo.u-bourgogne.fr/territoiresduvin/index.php?id=1753].

Sophie LIGNON-DARMAILLAC, « Les routes du vin, une géographie multidirectionnelle. La nouvelle alliance du tourisme et du vin ", dans Laurent Bourdeau et Pascale Marcotte (dir.), Les routes touristiques, Presses de l'Université Laval, 2015.

Sophie LIGNON-DARMAILLAC, «L'œnotourisme, redécouverte des valeurs patrimoniales des vignobles historiques, développement des vignobles du nouveau-monde ", Cultur (Revista de Cultura e Turismo), édition spéciale, année 8, n 3, 2014 [https://periodicos.uesc.br/index.php/ cultur/article/view/358/362].

Sophie LIGNON-DARMAILLAC, L'œnotourisme en France. Nouvelle valorisation des vignobles. Analyse et bilan, Féret, 2009.

Sophie LIGNON-DARMAILLAC, « Des terroirs à voir et à boire : les routes du vin méditerranéennes ", colloque international « Les terroirs : caractérisation, développement territorial et gouvernance », Aix-en-Provence, 2007.

Jacques MABY, «Paysage et imaginaire : l'exploitation de nouvelles valeurs ajoutées dans les terroirs viticoles ", Annales de géographie, tome 111, n 624, 2002 [https://doi.org/10.3406/geo. 2002.1666].

Pascale MARCOTTE, Laurent BOURDEAU et Maurice DOYON, « Agrotourisme, agritourisme et tourisme à la ferme. Une analyse comparative », Téoros, vol. 25, n³, 2006 [https:// journals.openedition.org/teoros/1091].

MEDIOBANCA, I canali di vendita del vino in Italia nel 2014 - indagine Mediobanca, I numeri del vino, 2014 [http://www.inumeridelvino.it/2015/03/].

OBSERVATOIRE RÉGIONAL DU TOURISME, Rapporto sul turismo in Toscana-La congiuntura 2018, Toscane, Bureau régional de statistique, 2019.

Bernard PECQUEUR, «Qualité et développement territorial : l'hypothèse du panier de biens et de services territorialisés » Économie rurale, vol. 261, n 1, 2001 [https://doi.org/10.3406/ecoru. 2001.5217].

Coline PERRIN et Filippo RANDELLI, « L'essor des viticulteurs étrangers dans le Chianti », colloque franco-britannique de géographie rurale de Vichy « Les étrangers dans les campagnes », Clermont-Ferrand, Presses universitaires Blaise Pascal, 2008.

Coline PERRIN, «L'agritourisme périurbain dans les collines de Toscane centrale », colloque international « Les agricultures péri-urbaines, un enjeu pour la ville : vers des projets de territoire », Université de Paris X Nanterre, École nationale supérieure du paysage de Versailles, 2010.

Marie-Claude PICHERY, « Patrimonialisation et stratégie d'œnotourisme », Territoires du vin, 2018 [http://preo.u-bourgogne.fr/territoiresduvin/index.php?id=1354].

Jean-Robert PITTE, « Le vin des brumes : note sur la renaissance de la viticulture dans les Îles Britanniques ", Hommes et Terres du Nord, numéro hors-série " Campagnes et littoraux d'Europe. Mélanges offerts à Pierre Flatrès », 1988 [https://doi.org/10.3406/htn.1988.3082]. 
Jean PONCET, La colonisation et l'agriculture européenne en Tunisie depuis 1881, Mouton, 1962.

Isabelle RABOUD-SCHÜLE, « Vacances et vin en Valais », dans Thomas Antonietti et Marie-Claude Morand (dir.), Mutations touristiques contemporaines : Valais 1950-1990, édition des Musées cantonaux du Valais, 1993 [http://doc.rero.ch/record/118002/files/BCV_NB_1796_3.pdf].

Filippo RANDELLI et Raphaël SCHIRMER, « Les vignobles du Chianti et de Bordeaux : deux destinations que tout différencie », colloque international des XXII Entretiens du Centre Jacques Cartier « Patrimoines alimentaires et destinations touristiques : passion ou raison », Lyon, 2009.

Hafedh SETHOM, L'agriculture de la presqu'île du Cap Bon (Tunisie), publication de l'Université de Tunis, 1977.

Mohamed souIssi, Valorisation touristique du paysage et du patrimoine rural et vitivinicole : l'exemple de la région du Cap Bon (Tunisie), actes du colloque international organisé par l'Université d'OrléansFrance « Paysages, mises en scènes paysagères, patrimoines vitivinicoles, valorisations touristiques et développement territorial », 2016.

Mohamed SOUISSI et Jean RIEUCAU, « L'œnovélotourisme, un tourisme vitivinicole lent, dans les vignobles du Cap Bon (Tunisie) et des Côtes du Rhône méridionales (France) », colloque international « Slow tourisme/slow sport : enjeux de spatialités, de mobilités et de corporéités », Université du Littoral-Côte d'Opale, Boulogne sur Mer-France, 2018.

UNION CENTRALE DES COOPÉRATIVES VITICOLES, Statistiques et rapport d'activité, Tunis, 2014.

VINTUR, « VADEMECUM de l'œnotourisme européen », 2005 [https://docplayer.fr/24917123-Projetvintur-vademecum-de-l-oenotourisme-europeen].

\section{NOTES}

1. Les terroirs sont perçus comme des espaces vivants et innovants spécialisés dans une production agricole qui, pour gérer leur ressource principale et préserver leur patrimoine et leur identité, ont besoin d'une multitude d'acteurs et d'une gouvernance particulière (Maby, 2002). De ce fait, le terroir viticole au Cap Bon est un espace viticole lisible qui se caractérise par une histoire, des procédés de fabrication et un vin qui lui sont propres. Il correspond donc à un ensemble rural dont l'unité est fondée sur une identité forte et un système socioéconomique homogène. Par contre, le territoire viticole de la région regroupe généralement des terroirs différents. Le territoire ajoute à l'espace des significations supplémentaires (une identité collective, un mode de découpage et de contrôle, des valeurs patrimoniales, une histoire sur la longue durée). Il témoigne donc d'une appropriation à la fois économique, idéologique et politique de l'espace (Di Méo, 1998).

2. En général, l'agritourisme a pour but de favoriser les produits locaux, de réutiliser les vieux bâtiments ruraux qui ne sont plus utilisables par l'agriculture moderne et d'augmenter le revenu des agriculteurs. Mais les pratiques ont évolué, l'agritourisme a pris de l'importance et son périmètre s'est agrandi. Des chercheurs avancent aujourd'hui le terme " agritourisme intégré ", pouvant se situer en différents lieux (pas uniquement dans les fermes), être sous la responsabilité de différents acteurs du territoire (pas uniquement les fermiers) et concerner une grande variété d'offres (pas uniquement les activités classiques) (Durrande-Moreau, Courvoisier et Bocquet, 2017).

3. Dans ce sens, le terme d'authenticité renvoie au territoire viticole qui peut être assimilé à un territoire-patrimoine chargé de valeurs héritées du passé contribuant à la définition des traditions et de l'identité locales. Souvent considéré par le plus grand nombre comme quasi- 
immuable dans ses formes paysagères, le territoire viticole devient alors le support de transmission de la culture des sociétés qui y ont vécu et qui y vivent peut-être encore (Delignières, 1998).

4. Le tourisme alternatif est l'opposé du tourisme conventionnel. Il se veut une alternative au tourisme de masse. Il englobe les nouvelles formes de tourisme qui ont pour objectifs de préserver les éléments qui composent l'identité du pays (culture, histoire, environnement) par une approche plus écologique, plus responsable et plus conforme au développement durable. Cette définition se rapproche de la définition du tourisme durable présentée dans la charte mondiale du tourisme durable de Lanzarote de 1995. Le tourisme alternatif s'adresse donc aux touristes qui veulent découvrir la Tunisie de façon différente, sans passer forcément par les stations touristiques balnéaires. C'est un tourisme respectueux des traditions, de la culture et du mode de vie des populations locales.

5. La patrimonialisation est le fait de donner un caractère patrimonial à un objet et peut être présentée comme un processus socioculturel par lequel un espace, un bien ou un objet (matériel), un concept ou une pratique (immatériel) sont transformés en un composant du patrimoine. Quelle que soit la nature de cet élément (naturelle, culturelle, ...), il devient un bien collectif à la fois objet de conservation et enjeu de protection, éventuellement de restauration (Pichery, 2018). 6. Une route ou un circuit touristique se définit comme un trajet à suivre pour atteindre une destination en passant par des sites touristiques ouverts aux visiteurs le long d'un chemin pittoresque, où des services sont disponibles. Il peut être appelé "circuit " si le trajet est en boucle, c'est-à-dire si le départ et l'arrivée se font au même point. Il sera appelé « route » si les points de départ et d'arrivée sont différents (Beaudet, 2003).

7. Le principe d'une route à thème consiste à créer un système de relations entre des patrimoines de même nature, afin de concevoir un produit capable de dynamiser l'économie touristique d'un territoire. Accroître la fréquentation, l'organiser et l'étaler dans le temps, tout en suscitant des retombées économiques directes et indirectes le long de l'itinéraire, valoriser un patrimoine, trouver des débouchés supplémentaires pour les producteurs, allonger la durée des séjours... (Carlier, 2000).

8. En France, un premier musée du vin a été ouvert à Colmar en 1927, bien avant la création de la route des vins à laquelle il s'intègre aujourd'hui. En 1931, c'est en Champagne que des collections consacrées au vin sont présentées au musée d'Épernay. Progressivement, après la Seconde Guerre mondiale, chaque vignoble aménage son propre musée du vin, chaque fois dans un lieu exceptionnel, représentatif de la région.

9. Le terme de patrimoine gastronomique renvoie à l'ensemble des éléments gastronomiques, matériels ou immatériels (produits, recettes ou savoirs et savoir-faire culinaires ancrés dans la mémoire sociale) caractérisant un héritage collectif territorial (Bessière, 2000).

10. L'Italie est, avec l'Espagne et la France, l'un des trois principaux pays viticoles au monde, tant par la surface et la diversité de ses vignobles, que par leur histoire ou le volume de leur production. L'Italie est le seul pays au monde à avoir, dès 1985, promulgué une loi spéciale sur l'agritourisme (Randelli et Schirmer, 2009).

11. Cette situation de premier espace viticole européen à avoir été mis en tourisme s'explique par le caractère ancien de la mise en valeur touristique des produits locaux et de la gastronomie régionale en Italie. D'ailleurs, dès les années 1930, le régime fasciste italien encourageait la consommation de produits nationaux en relançant la visite des régions italiennes par la construction de voies ferrées. Un guide, La Guida gastronomica d'Italia, inventoriait et localisait les plats et les vins typiques pour chaque région (Lignon-Darmaillac, 2007).

12. Le Chianti est un terroir italien, c'est-à-dire un espace économique homogène spécialisé dans la production d'un vin. Il s'identifie à l'aire de l'appellation viticole Chianti Classico, délimitée par décret ministériel en 1932 et restée inchangée depuis. L'aire s'étend sur quatre communes de la 
province de Sienne (centre de l'Italie) et quatre communes de la province de Florence (capitale de la Toscane).

13. L'agritourisme est un phénomène ancien dans les vignobles du Chianti. Dès 1947, une Suisse a transformé un hameau de Castellina in Chianti en chambres d'hôtes pour étrangers, avec succès jusqu'aujourd'hui. Son exemple a été suivi par plusieurs investisseurs allemands et britanniques (Perrin, 2010).

14. Le séjour dans l'exploitation permet ici une rencontre culturelle entre ruraux et citadins. Le propriétaire passe du temps avec ses hôtes, leur donne des conseils d'excursions (visite de la ferme et du musée local du vin, organisation de veillées de chansons et de contes, etc.).

15. Le Cap Bon est le principal producteur d'agrumes en Tunisie : il en met en vente près de 300000 tonnes par an sur le marché (contre 54000 tonnes produites dans le reste du pays), cueillis sur une superficie de plus de 20000 hectares situés surtout sur le flanc-est de l'épine dorsale du Cap, dans les espaces agricoles de Menzel Bouzelfa et de Béni Khalled (Union centrale des coopératives viticoles, 2014).

16. Dès 1987, Grombalia est déclarée ville internationale de la vigne et du vin lors des assises de l'office international de la vigne et du vin à Rome.

17. La production de vin tunisienne se situe autour de 600000 hectolitres selon les années. Ce pays bénéficie d'un climat particulièrement favorable au développement de cette activité, facteur qui, avec son sol calcaire-limoneux, permet de cultiver la vigne dans des conditions idéales. Ses vins sont commercialisés dans la plupart des pays européens et sont particulièrement appréciés en France et en Italie.

18. En 1902, les statistiques officielles ont montré, qu'il y avait, en Tunisie, une importante population italienne, principalement sicilienne, composée d'environ 17000 agriculteurs travaillant pour leur propre compte et de presque 4000 journaliers agricoles, ouvriers défricheurs, charbonniers, vignerons, etc. (Jacueton, 1902).

19. Autour des années 1930, le château Saint-Augustin constituait une ferme pilote, où les étudiants français de l'école coloniale de Tunis venaient faire leur stage bisannuel, afin d'acquérir les connaissances pratiques du secteur de la viticulture et de la fabrication du vin.

20. À ce jour, il n'existe aucune démarche de patrimonialisation officielle du patrimoine viticole colonial en Tunisie. Les actions menées en faveur de la conservation, de la protection et de la restauration du patrimoine sont le fruit d'initiatives privées. Elles sont généralement menées par des particuliers dans un domaine familial.

21. Depuis sa création en 1932, la cave de Bir Drassen est un haut lieu du vin tunisien, ayant développé un savoir-faire reconnu au fil de son histoire, en particulier dans la vinification et l'élevage des vins rouges, grâce à des équipements et un chai souterrain parfaitement adapté à l'élevage en barrique. En 1954, la cave a obtenu une médaille d'argent au concours général agricole de Paris.

22. À partir de cette date, le Cap Bon a attiré une partie des réfugiés andalous chassés lors de la reconquête de l'Espagne. Ceux-ci importèrent de nouvelles techniques agraires, renforçant le caractère agricole de la région.

23. Du fait de l'absence d'études et de statistiques officielles sur la fréquentation touristique des fermes viticoles, notre analyse sera fondée sur les résultats de nos enquêtes de terrain auprès des propriétaires de caves et des différents groupes d'acteurs locaux.

24. «Envie de Voyages », producteur sur l'Europe et la France, installé à Auray en Bretagne, est l'exemple d'un tour-opérateur qui propose des circuits viticoles dans la région du Cap Bon. De son côté, le club privé en ligne "Voyage Privé », spécialiste de la vente de voyage haut de gamme, propose à ses membres des circuits « route des vins » dans les vignobles du Cap Bon.

25. En plus des circuits locaux, des professionnels tunisiens et italiens ont inauguré le 7 mars 2018 le circuit viticole «Magon » entre le Cap Bon en Tunisie et la Sicile en Italie. Ce nouveau circuit culturel transfrontalier cofinancé par l'Union européenne vise à valoriser le patrimoine et 
l'origine commune du vin entre les deux rives de la Méditerranée. Tous les sites intégrant ce circuit ont été aménagés, avec l'ajout d'une signalétique spécifique à la route Magon. Une vingtaine de guides ont également été formés pour accompagner les touristes.

26. Le tourisme rural est une forme de tourisme alternatif qui englobe l'ensemble des activités touristiques pratiquées dans le territoire rural humanisé. Il est aussi associé à une expérience récréative dont les activités ne se retrouvent pas en milieu urbain et de nature agricole. Le tourisme à la ferme et l'agrotourisme sont ainsi considérés comme des composantes du tourisme rural, domaine auquel ils doivent s'intégrer et participer (Marcotte, Bourdeau et Doyon, 2006).

\section{RÉSUMÉS}

Le développement de l'œnotourisme et la possibilité d'intégrer la dimension paysagère et patrimoniale dans les activités touristiques placent les vignobles de la péninsule du Cap Bon (Tunisie) en excellente position pour associer terroir, patrimoine et paysage. La mise en tourisme du patrimoine viticole et des ressources naturelles non liées exclusivement au soleil et à la mer trouvent ainsi un nouveau sens (gastronomie locale, produits de terroirs, routes viticoles, labellisation). Malgré l'importance des aménités touristiques viticoles, l'œnotourisme au Cap Bon ne représente pas encore une réelle offre alternative, ou du moins, sa promotion n'est pas ou peu visible. L'objet de cet article est de comprendre l'émergence d'une fréquentation œnotouristique à partir d'entretiens avec des acteurs locaux. Il vise également à démontrer dans quelles conditions le Cap Bon pourrait intégrer la valorisation de son paysage et de son patrimoine viticole colonial aux politiques de diversification du produit touristique tunisien.

Development of wine tourism and the possibility of integrating landscape and heritage dimension into tourist activities would allow the vineyards of Cap Bon (Tunisia) to have an excellent position to associate terroir, heritage and landscape. Taking wine tourism heritage and natural resources not linked solely to assets of sunshine and the sea would give them, therefore, a new sense (local gastronomy and products, wine routes, labeling). Despite, the importance of wine tourism amenities, wine tourism in Cap Bon is not yet an alternative offer, or at least, its promotion is not, or is hardly, visible. The issue of this paper is to understand the emergence of wine tourism through interviews with local actors. Also, it aims to demonstrate the conditions in which the Cap Bon may integrate the valorization of its landscape and its wine heritage of the colonial period into the diversification policy of Tunisian tourism.

\section{INDEX}

Mots-clés : œnotourisme, patrimonialisation, vignobles, Cap Bon, Tunisie

Keywords : wine tourism, heritage, vineyards, Cap Bon, Tunisia 


\section{AUTEUR}

\section{MOHAMED SOUISSI}

Maître-assistant, chercheur spécialisé en tourisme et développement territorial

Université de Sfax (Tunisie), Laboratoire Syfacte

mohamedsouissi2114[at]yahoo.fr 